\title{
Detection of Schmallenberg virus RNA in bull semen in Poland
}

\author{
J. Kęsik-Maliszewska, M. Larska \\ Department of Virology, National Veterinary Research Institute, \\ Al. Partyzantów 75, 24-100 Puławy, Poland
}

\begin{abstract}
The detection of Schmallenberg virus (SBV) in the breeding bull semen raised the question of the possibility of venereal transmission of SBV which could result in cost-intensive restrictions in the trade of bovine semen. In order to evaluate the presence of SBV RNA in bovine semen, 131 bull semen samples from four locations in Poland collected between 2013 and 2015 were analysed by RT-PCR for viral RNA. SBV RNA was detected in 5.3\% of the samples. The study has revealed that application of an appropriate RNA extraction method is crucial to detect virus excretion via semen.
\end{abstract}

Key words: Schmallenberg virus, bull, semen, extraction method

\section{Introduction}

Schmallenberg virus is a new Orhtobunyavirus which has been recently identified in Europe causing fever, milk drop and congenital abnormalities in ruminants (Hoffman et al. 2012). Several studies have confirmed the shedding of SBV by RT-PCR in up to $11.6 \%$ of bovine semen samples tested (Hoffman et al. 2013). Despite the fact, that the possibility of venereal route of SBV transmission has not been shown, the infectivity of SBV in semen has been confirmed by subcutaneous inoculation of naïve heifers and interferon $\alpha / \beta$ receptor-deficient mice (Schulz et al. 2014). SBV epidemic has caused cost-intensive trade restrictions, especially in the bovine semen trade.

The first cases of SBV in Poland have been detected in the summer of 2012. The virus has spread rapidly throughout the country infecting over $34 \%$ ruminants, with up to $92 \%$ seroprevalence at the prov- ince level in 2013 (Larska et al. 2014). The aim of this study was to assess the presence of the virus in the semen of Polish bulls.

\section{Materials and Methods}

Commercially diluted semen samples aliquoted in straws were collected between 2013 and 2015. The samples originated from 131 breeding bulls in the age of 1.5 up to 6.5 years from four herds located in Opolskie, Warmińsko-Mazurskie, Wielkopolskie and Zachodniopomorskie provices. The serological status of individual animals could not be determined. The sensitivity and efficiency of the extraction method were assessed using DNA standard prediluted in semen. RNA from SBV positive brain homogenate was extracted, RT-PCR product was electrophoretically separated, the specific amplicon (SBV-S) of $87 \mathrm{bp}$ was 


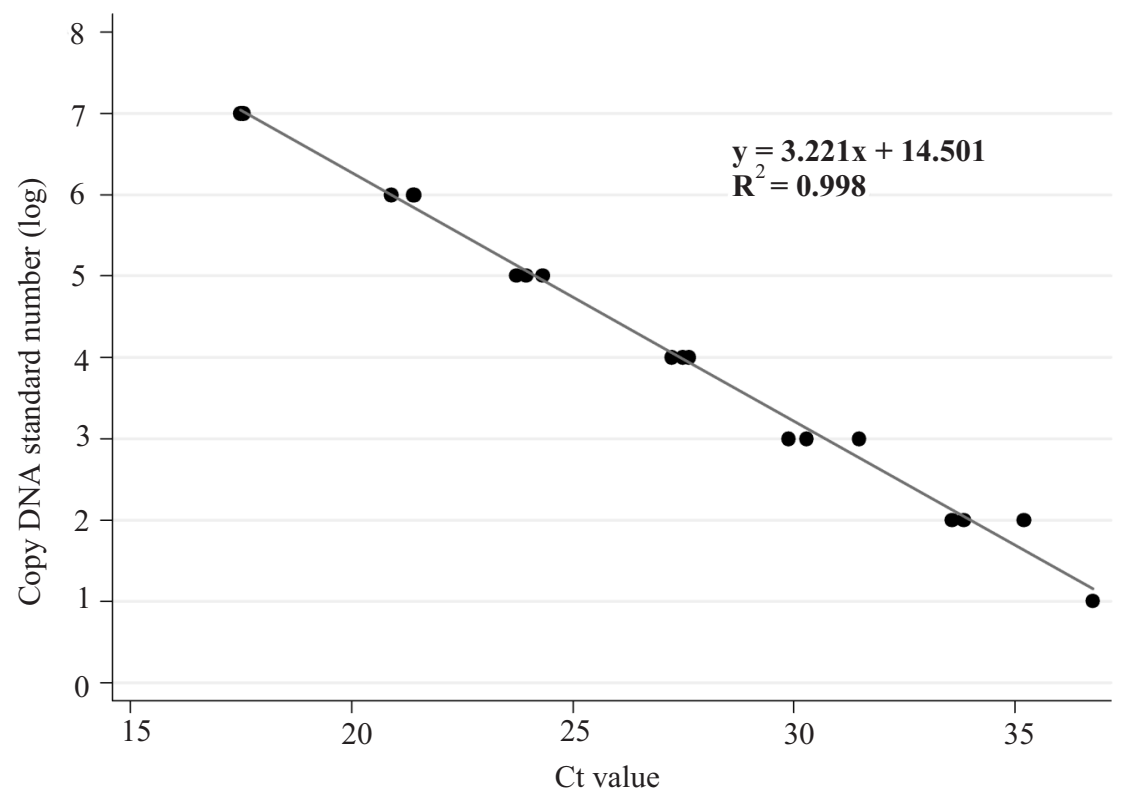

Fig. 1. Regression curve of tenfold semen dilutions of DNA standard of SBV S segment fragment. Each dilution was tested three times (represent by the scatter).

cut out from the gel and purified. DNA concentration was measured and the number of SBV-S copies was calculated. Tenfold dilutions of DNA standard in TE buffer and semen diluted in egg yolk-free extender (commercially available from a semen bank, containing seminal fluid with spermatozoa, confirmed as SBV negative by RT-PCR and 3 subsequent passages in BHK-21) were prepared. Since the extraction method is critical for the detection of SBV in semen (Schulz et al. 2015), four extraction methods were assessed for the level of $\beta$-actin (internal control of amplification) and recovery rate of the DNA standard in semen. Three of them were commercial manual silica membrane-based methods: RNeasy Mini Kit (Qiagen); Viral RNA Mini Kit (Qiagen) preceded by lysis with Qiazol Lysis Reagent (Qiagen, 2013); High Pure Viral Nucleic Acid Kit (Roche); while the forth was TRI Reagent RNA extraction. Real-time RT-PCR was performed using SBV-S and bovine $\beta$-actin specific primers and AgPath-ID One-Step RT-PCR Reagents kit (Ambion, Applied Biosystem) in Step One Real-Time PCR system (Life Technologies).

\section{Results and Discussion}

Qiazol lysis followed by Viral RNA Mini Kit (Qiagen) extraction was selected as the most suitable extraction method allowing the detection of SBV RNA in the spiked semen at the limit of 10 copies per reaction and PCR efficiency of $99.8 \%$ (Fig. 1). The $\beta$-actin $C_{t}$ (threshold cycle) values for the extracted bovine RNA were by average of 2 cycles lower (mean
$C_{t}$ 36.0) than those obtained by other extraction methods, which suggested that Viral RNA Mini kit was the most efficient test in our hands. Lower detection limit of 1 copy per reaction and the efficiency of 93\% was established for DNA standard diluted in TE buffer. This demonstrates that the semen has an inhibitory effect on SBV detection and emphasizes the proper test selection to improve the detectability of bulls shedding SBV in the semen (Schulz et al. 2015).

Seven semen samples tested in duplicate out of 131 samples $(5.3 \%)$ gave positive real-time RT-PCR results with $C_{t}$ values between 34.5 and 37.1. The positive samples were collected at the end of 2013 and in 2014 , originated from two herds where the in-herd prevalence were $4.8 \%$ and $13.3 \%$. The occurrence of SBV in the semen of Polish bulls may be explained by the recent circulation of the virus as suggested by the observed increase in seroprevalence among Polish cattle between 2012 when first SBV bovine cases were reported and 2013 when the overwintered virus continued to spread (Larska et al. 2014). The observed proportion of SBV RNA positive semen and Ct values are consistent with results obtained in the studies from Germany, France and Netherlands (Hoffmann et al. 2013, Van der Poel et al. 2013, Ponsart et al. 2014). SBV excretion should be considered as an important risk factor for SBV transmission, since Schulz et al. (2014) have demonstrated viremia and seroconversion of a heifer after subcutaneous infection using a bull semen with relatively high $\mathrm{C}_{\mathrm{t}}$ of 34.2.

We have also demonstrated that the detection of SBV in the semen required validation of an appropriate RNA extraction method and sample preparation. 
RNA recovery from the tested semen samples was the most efficient using the modified with Qiazol Lysis reagent Viral RNA Mini kit. The differences in the sensitivities of RNA extraction methods between our study and the German interlaboratory comparisons, where Trizol-based methods were superior (Hoffmann et al. 2013, Schultz et al. 2015) may be connected to the fact that Polish semen samples came as already prediluted in the straws. Besides, different semen extenders may have also the inhibitory effect on PCR and therefore, they may influence SBV detection (Van Der Poel et al. 2014).

In conclusion, these results emphasize the importance of monitoring of bull semen for the presence of SBV as a potential source of infection as well as the need for improved diagnostic tools. The infection of bulls between 2013 and 2014 confirms ongoing circulation of SBV in the country. The potential venereal transmission of SBV trough semen could lead to new outbreaks of disease in naïve herds, even during intra-seasonal absence of vector. Artificial insemination, besides the vector movement and animal transportation, may have also increased the observed rapid spread of SBV inside the country, and it might have been the source of distant outbreaks during first epidemic wave. The relatively low proportion of SBV contaminated semen in Poland should not be underestimated, since one ejaculate produces approx. 40 semen straws, therefore one false negative or one untested semen sample may be potentially responsible for infecting the same number of naïve animals. Furthermore, the pattern of persistent shedding of SBV in the semen of infected bulls (even three months post infection, Ponsart et al. 2014) may contribute to prolonged risk of SBV transmission. The study highlights the need for further research of SBV infection in males, particularly in relation to SBV spread as well as to the impact of the infection on semen quality. The latter being a consequence of fever accompanying the viremia and the inflammatory process in the presence of SBV in spermatozoa and lymph nodes (Hoffman et al. 2013, Van Der Poel et al. 2014).

\section{Acknowledgments}

We would like to thank to Áine Collins (Teagasc) for English corrections and Prof. Mirosław P. Polak (NVRI) for his helpful comments. The work was funded by the National Centre of Research and Development (NCBiR; project No PBS2/A8/24/2013).

\section{References}

Hoffmann B, Schulz C, Beer M (2013) First detection of Schmallenberg virus RNA in bovine semen, Germany, 2012. Vet Microbiol 167: 289-295.

Larska M, Kęsik-Maliszewska J, Kuta A (2014) Spread of Schmallenberg virus infections in the ruminants in Poland between 2012 and 2013. B Vet I Pulawy 58: 169-176.

Ponsart C, Pozzi N, Bréard E, Catinot V, Viard G, Sailleau C, Viarouge C, Gouzil J, Beer M, Zientara S, Vitour $\mathrm{D}$ (2014) Evidence of excretion of Schmallenberg virus in bull semen. Vet Res 45: 37.

Qiagen (2013) User-Developed Protocol For manual purification of SBV RNA from bull semen: https://www.qiagen.com/fo/resources/download.aspx?id= 64f26086-2199-4a4a-b5ec-ee10a6367228\&lang=en

Schulz C, van der Poel WH, Ponsart C, Cay AB, Steinbach F, Zientara S, Beer M, Hoffmann B (2015) European interlaboratory comparison of Schmallenberg virus (SBV) real-time RT-PCR detection in experimental and field samples: The method of extraction is critical for SBV RNA detection in semen. J Vet Diagn Invest 27: 422-430.

Schulz C, Wernike K, Beer M, Hoffmann B (2014) Infectious Schmallenberg virus from bovine semen, Germany. Emerg Infect Dis 20: 338-340.

Van Der Poel WH, Parlevliet JM, Verstraten ER, Kooi EA, Hakze-Van Der Honing R, Stockhofe N (2014) Schmallenberg virus detection in bovine semen after experimental infection of bulls. Epidemiol Infect 142: 1495-1500. 